\title{
Evaluación de cuatro genotipos de pepino tipo largo cultivados bajo ambiente protegido*
}

\author{
Evaluation of four long size cucumber genotypes grown under greenhouse conditions \\ José Aníbal Cruz-Coronado ${ }^{1}$ \\ José Eladio Monge-Pérez ${ }^{2}$
}

Fecha de recepción: 29-5-19

Fecha de aceptación 16-3-21

\begin{abstract}
Resumen
El objetivo del estudio fue evaluar el rendimiento y la calidad de cuatro genotipos de pepino (Cucumis sativus L.) partenocárpico de tipo largo (Arioso, Cumlaude, Kalunga y Paisaje), cultivados bajo condiciones de invernadero en la Estación Experimental Agrícola Fabio Baudrit Moreno, en Alajuela, Costa Rica. El cultivo se manejó con fertirrigación en sustrato de fibra de coco y con poda a un tallo por planta.

En cuanto a los datos, muestran una amplia variabilidad entre los genotipos en edad al inicio de floración (19 - 21 días después del trasplante-ddt), edad al inicio de cosecha ( 28 - $30 \mathrm{ddt})$, longitud del fruto $(31,02-32,38 \mathrm{~cm})$, diámetro del fruto $(48,74-52,31$ $\mathrm{mm})$, número total de frutos por planta $(16,6 \mathrm{o}-26,92)$, peso del fruto $(470,17-517,77 \mathrm{~g})$, rendimiento comercial $(14,47$ - 23,53 $\mathrm{kg} / \mathrm{m} 2)$, rendimiento total $(20,49-33,46 \mathrm{~kg} / \mathrm{m} 2)$ y porcentaje de sólidos solubles totales (3,01 - 3,64 $\left.{ }^{\circ} \mathrm{Brix}\right)$.

Los frutos de pepino de primera calidad mostraron mayor longitud y diámetro que los de segunda calidad y de rechazo. En términos generales, los frutos de primera y segunda calidad obtuvieron un mayor porcentaje de sólidos solubles totales que los de calidad de rechazo. El genotipo Cumlaude obtuvo el mayor rendimiento de primera calidad $(12,61 \mathrm{~kg} / \mathrm{m} 2)$, por lo que se considera el mejor adaptado a las condiciones en las que se desarrolló la investigación; por el contrario, Arioso obtuvo el menor rendimiento comercial y total.

Palabras clave: Cucumis sativus, genotipos, pepino, invernadero, partenocárpico, rendimiento, longitud del fruto, diámetro del fruto, peso del fruto, calidad
\end{abstract}

\begin{abstract}
The objective of the study was to evaluate yield and quality of four long fruit size parthenocarpic cucumber (Cucumis sativus L.) genotypes (Arioso, Cumlaude, Kalunga and Paisaje) grown under greenhouse conditions at the Agricultural Experimental Station Fabio Baudrit Moreno located in Alajuela, Costa Rica. The crop was planted on coconut fiber as substrate, pruned to one stem per plant and managed with fertigation. Data show a wide variety among genotypes with respect to days to start of flowering (19-21 days after transplant -dat-), days to start of harvest ( 28 - 30 dat), fruit length $(31,02-32,38 \mathrm{~cm})$, fruit diameter $(48,74-52,31$ $\mathrm{mm}$ ), total number of fruits per plant $(16,6 \mathrm{o}-26,92)$, fruit weight ( $470,17-517,77 \mathrm{~g})$, commercial yield $(14,47-23,53 \mathrm{~kg} / \mathrm{m} 2)$, total yield $(20,49-33,46 \mathrm{~kg} / \mathrm{m} 2)$, and percentage of total soluble solids (3,01 - 3,64 $\left.{ }^{\circ} \mathrm{Brix}\right)$. First quality cucumber fruits showed higher length and diameter than second quality and waste quality fruits. In general, first and second quality fruits obtained higher percentage of total soluble solids than waste quality fruits. Cumlaude genotype obtained the highest first quality yield (12,61 kg/m2), so it is considered the best adapted to the conditions of the trial; otherwise, Arioso obtained the lowest commercial and total yield. Key words: Cucumis sativus, genotypes, cucumber, greenhouse, parthenocarpic, yield, fruit length, fruit diameter, fruit weight, quality

1 *Este trabajo forma parte de la tesis de licenciatura en Agronomía del primer autor, Sede de Guanacaste, Universidad de Costa Rica. Costa Rica. Licenciado en Agronomía, Universidad de Costa Rica. Correo electrónico: cruzcsanibal@gmail.com

2 Máster en Ciencias Agrícolas y Recursos Naturales, Docente de la Sede de Guanacaste e Investigador de la Estación Experimental Agrícola Fabio Baudrit Moreno, Universidad de Costa Rica. Costa Rica. Correo electrónico: melonescr@yahoo.com.mx
\end{abstract}




\section{Introducción}

El pepino Cucumis sativus L., pertenece a la familia de plantas cucurbitáceas, y es una hortaliza que se cultiva en condiciones tropicales y subtropicales alrededor del mundo. Esta es una especie nativa del norte de India (Kapuriya, y otros, 2017), la planta es herbácea, anual, de hábito rastrero o trepador cuando se le proporciona un tutorado, y cuyo crecimiento es indeterminado (Reche, 2011). Sus frutos se consideran una buena fuente de minerales y vitaminas, tales como: calcio, cloro, potasio, hierro, vitamina $\mathrm{C}$, y pequeñas cantidades del complejo vitamínico B (Sarhan \& Ismael, 2014; Rozano, Quiróz, Acosta, Pimentel, \& Quiñones, 2004).

En Costa Rica, las variedades de pepino más utilizadas por los productores eran: Dasher II, Poinsett 76 y General Lee, debido al buen resultado productivo que presentaban (Torres, 2007). Sin embargo, siempre es conveniente invertir recursos en investigación sobre variedades y productos hortícolas innovadores y de altos rendimientos, que ofrezcan opciones más rentables a los agricultores (Monge-Pérez, 2016).

Según el Programa Nacional Sectorial de Producción Agrícola Bajo Ambientes Protegidos (PRONAP), entre 2008 y 2009, Costa Rica contaba con 13 ha de producción bajo ambiente protegido, dedicadas al cultivo de cucurbitáceas (Marín, 2010), entre ellas el pepino.

El cultivo de pepino en Costa Rica está distribuido en varias zonas del país y está dirigido principalmente a satisfacer la demanda nacional. Sin embargo, entre los años 2007 y 2010, se desarrolló un proyecto de producción de pepino holandés para exportación en la zona de Zarcero; la poca experiencia en producción para exportación afectó dicho proyecto, el cual fracasó debido a varias razones, entre las que destacan la poca investigación sobre cultivares promisorios (Mora, Campos, Fernández, \& Morales, 2007), así como la baja productividad y rentabilidad alcanzadas (Valenciano, Salas, \& Díaz, 2013).

Asimismo, la producción de pepino en Costa Rica fue de 1500 toneladas métricas (ton) en 2010, pero bajó a 1000 ton en el 2011-2012 y a 940 ton en 2013. Dicha producción representó un rendimiento promedio de 9,1 ton/ha en 2010, 2011 y 2012, esta cayó a 8,9 ton/ha en 2013 (FAO, 2015).

Las condiciones óptimas de temperatura para el desarrollo del pepino se encuentran en un rango de 18 a $28{ }^{\circ} \mathrm{C}$ (Bojacá \& Monsalve, 2012). Por otra parte, la FAO indica que el pepino se desarrolla de manera óptima en un rango de temperatura entre 18 a $25{ }^{\circ} \mathrm{C}$ y una humedad relativa entre 70 y $90 \%$ (FAO, 2002).

Por ello, el pepino se adapta a climas cálidos y templados, este se cultiva desde las zonas costeras hasta los 1200 metros sobre el nivel del mar. Por debajo de $14{ }^{\circ} \mathrm{C}$, y por encima de $40{ }^{\circ} \mathrm{C}$, el crecimiento se detiene; por lo tanto, es una planta con altos requerimientos de humedad, por lo que la humedad relativa óptima durante el día es de 60 a $70 \%$, y durante la noche de 70 a $90 \%$ (Casaca, 2005).

Las temperaturas muy altas durante el periodo de floración disminuyen la viabilidad del polen (Haifa 2011), lo que afecta la producción. Asimismo, se considera que las temperaturas de 20 a $30{ }^{\circ} \mathrm{C}$ durante el día apenas tienen incidencia sobre la producción; a mayor temperatura, hasta $25{ }^{\circ} \mathrm{C}$, se alcanza la máxima producción precoz, pero por encima de $30{ }^{\circ} \mathrm{C}$ se pueden observar desequilibrios en las plantas (Vasco, 2003).

Cabe destacar que el pepino posee varios cultivares en el mercado, con diferente tamaño, forma, coloración de los frutos, textura de la cáscara, sabor y características vegetativas (Crosby, 2008). Según diversos autores, se identifican cinco grupos: pepino para ensalada, tipo caipira, tipo japonés, tipo holandés, y tipo industrial (para conserva) (Sediyama, Nascimento, Lopes, Lima, \& Vidigal, 2014). Otros investigadores informan que los tipos más comunes de pepino son: americano, europeo, del este medio, holandés, y oriental (López-Elías, y otros, 2015).

Con respecto a la clasificación de los pepinos, una de ellas se basa en las características físicas de los frutos: pepino tipo español, con frutos de longitud máxima de $15 \mathrm{~cm}$, de color verde y que se usan en encurtido o consumo fresco; el pepino tipo francés, con frutos entre 20 a $25 \mathrm{~cm}$ y con espinas en la cáscara y, por último, el pepino tipo holandés 
o Almería, con frutos de más de $25 \mathrm{~cm}$ de longitud, con plantas ginoicas de frutos totalmente partenocárpicos y de cáscara lisa, y de superficie algo asurcada (Centro de Exportación e Inversión de la República Dominicana, 2008).

Según su uso, los pepinos se pueden clasificar en: pepino para encurtido (de procesamiento), pepino de mesa o para ensalada (o de consumo fresco), cultivares de invernadero, armenio, oriental (japonés o asiático), Beit Alfa (persa) y europeo (Haifa, 2011). De la misma manera, otra de las clasificaciones utiliza como criterio el origen, como es el caso de los tipos holandés y francés (también llamados europeos) y el tipo asiático. Un criterio adicional es el tamaño del fruto: largo (tipo holandés), mediano (tipo americano o "slicer", y francés), también el pequeño (tipo Beit Alpha, mini, o pepinillo).

En cuanto a los pepinos de tipo holandés, destacan por ser de sabor suave, sin semillas y se cosechan de 30 a $36 \mathrm{~cm}$ de longitud. Además, su cáscara es delgada, lisa y sin espinas, con una excelente calidad comestible, por lo que no requieren de pelado para su consumo (Crosby, 2008). Este tipo de pepino se cultiva principalmente en invernadero (Johnny's Selected Seeds, 2014; Grijalva, Macías, Grijalva, \& Robles, 2011).

Por otra parte, los pepinos tipo asiático son espinosos y muy largos (Johnny's Selected Seeds, 2014).

Cabe destacar que el pepino es una hortaliza de alta demanda en países desarrollados, principalmente en la industria de vegetales procesados o para encurtido e incluso para consumo fresco (Musmade \& Desai, 2003). Por lo tanto, es importante evaluar nuevos materiales de este fruto que les sirvan a los productores para mantenerse en el mercado nacional e incluso incursionar en la actividad de exportación, con variedades vigorosas, precoces, de altos rendimientos y de buena calidad.

Entre los aspectos más importantes por considerar en la selección de genotipos de pepino destacan: rendimiento, crecimiento de la planta, adaptación a diferentes ambientes, resistencia a enfermedades y uniformidad de la cosecha (Vasco, 2003).
Por consiguiente, el objetivo de esta investigación fue evaluar el rendimiento y la calidad de cuatro genotipos de pepino partenocárpico de tipo largo, cultivados bajo ambiente protegido en condiciones hidropónicas, en Alajuela, Costa Rica.

\section{Materiales y métodos}

Se realizó la siembra de cuatro genotipos híbridos de pepino (Cucumis sativus L.) partenocárpico, con una longitud del fruto larga (cuadro 1). Este cultivo se realizó en condiciones hidropónicas en el invernadero de Hortalizas de la Estación Experimental Agrícola Fabio Baudrit Moreno (EEAFBM), ubicada en Barrio San José de Alajuela, Costa Rica, a una altitud de 883 msnm.

\section{Cuadro 1. Genotipos de pepino utilizados en el ensayo.}

\begin{tabular}{|l|l|}
\hline Genotipo & Casa comercial \\
\hline Arioso & Known-You Seed \\
\hline Cumlaude & Rijk Zwaan \\
\hline Kalunga & Enza Zaden \\
\hline Paisaje & Rijk Zwaan \\
\hline
\end{tabular}

Fuente: elaboración propia.

El cultivo se realizó en sacos plásticos rellenos con fibra de coco de $1 \mathrm{~m}$ de largo, $20 \mathrm{~cm}$ de ancho y $15 \mathrm{~cm}$ de altura, la distancia de siembra fue de $25 \mathrm{~cm}$ entre plantas, y de 1,54 m entre hileras, para una densidad de 2,6o plantas/ $\mathrm{m}^{2}$. Asimismo, las plantas se manejaron a un solo tallo, se eliminaron todos los tallos secundarios; las labores de amarre de la planta, deshijas y deshojas se realizaron en forma periódica, además, los primeros cuatro frutos de cada planta fueron desechados con el fin de lograr una cosecha más uniforme.

Durante este ensayo, las condiciones ambientales dentro del invernadero se dieron de la siguiente manera: la temperatura diurna promedio fue de $27,1^{\circ} \mathrm{C}$ con un valor mínimo promedio de $20,7^{\circ} \mathrm{C}$ y un máximo promedio de $33,4{ }^{\circ} \mathrm{C}$, mientras que la temperatura nocturna promedio de $21,4{ }^{\circ} \mathrm{C}$ con un valor mínimo promedio de $19,8{ }^{\circ} \mathrm{C}$ y un máximo promedio de $23,9{ }^{\circ} \mathrm{C}$. A su vez, la 
humedad relativa promedio durante el día fue de $72,3 \%$ y durante la noche de 89,2 \%. En cuanto a la radiación fotosintéticamente activa diurna promedio se dio de 494,0 $\mathrm{W} / \mathrm{m}^{2}$.

Con respecto al almácigo, se sembró el 3 de julio de 2015, para ello se usaron bandejas de 98 celdas y una turba ("peat moss") como sustrato. El trasplante se realizó 12 días después de la siembra, cuando las plántulas tenían una hoja verdadera y su período de cultivo abarcó desde el 15 de julio hasta el 20 de octubre de 2015, es decir, hasta los 97 días después del trasplante (ddt).

A su vez, el suministro hídrico y la fertilización se hicieron mediante un sistema de fertirriego automático controlado por un dispositivo marca iGrow 1400 (El plan de fertirriego se presenta en el cuadro 2).

En efecto, se clasificó la cosecha según tres categorías de calidad (cuadro 3).

\section{Cuadro 2. Plan de fertirriego utilizado en el ensayo.}

\begin{tabular}{|l|l|l|l|l|l|l|l|l|l|l|l|l|}
\hline \multicolumn{2}{|l|}{ Concentración del nutriente $(\mathrm{mg} / \mathrm{l})$} \\
\hline Etapa del cultivo & N & $\mathbf{P}$ & K & Ca & Mg & S & Cu & Fe & Zn & Mn & Mo & B \\
\hline 0-15 ddt & 150 & 53 & 240 & 165 & 40 & 50 & 0,16 & 2,9 & 0,3 & 0,6 & 0,9 & 0,8 \\
\hline $15-30 \mathrm{ddt}$ & 161 & 53 & 265 & 175 & 50 & 50 & 0,16 & 2,9 & 0,3 & 0,6 & 0,9 & 0,8 \\
\hline 30 ddt en adelante & 172,5 & 53 & 290 & 175 & 55 & 50 & 0,16 & 2,9 & 0,3 & 0,6 & 0,9 & 0,8 \\
\hline
\end{tabular}

Fuente: elaboración propia.

Cuadro 3. Parámetros de calidad de pepino utilizados en el ensayo.

\begin{tabular}{|l|l|l|l|}
\hline \multicolumn{4}{|l|}{ Categoría de calidad } \\
\hline Parámetro & Primera & Segunda & Rechazo \\
\hline Deformación de frutos & Ausente & Leve & Severa \\
\hline Daños en la cáscara del fruto & Ausente & Menor o igual a $1 \mathrm{~cm} 2$ & Mayor a $1 \mathrm{~cm} 2$ \\
\hline
\end{tabular}

Fuente: elaboración propia.

Para ello, se evaluaron las siguientes variables:

a) Edad al inicio de floración (ddt): esta variable se midió en días después del trasplante, cuando el $50 \%$ de las plantas mostraban al menos una flor completamente abierta.

b) Edad al inicio de cosecha (ddt): cuyo registro se dio días después del trasplante, al realizar la primera cosecha, cuando los frutos habían alcanzado un estado de desarrollo óptimo para la comercialización.

c) Presencia de espinas: se determinó en forma cualitativa en cada genotipo mediante las siguientes categorías: ausencia de espinas; cantidad intermedia de espinas; muchas espinas. d) Número de frutos por planta: se contabilizó el número total de frutos producidos por parcela y se dividió entre el número de plantas del terreno.

e) Longitud del fruto (cm): dicha característica se evaluó en 20 frutos de cada categoría de calidad en cada repetición y se obtuvo el promedio; para ello, se determinó con una cinta métrica marca Assist 32G-8025, con una capacidad de 800,o $\pm 0,1 \mathrm{~cm}$.

f) Diámetro del fruto $(\mathrm{mm})$ : fue medido en la parte media de 20 frutos de cada categoría de calidad por repetición y se obtuvo el promedio; para dicho fin, se midió con un calibrador digital marca Mitutoyo modelo CD-6"CS, con una capacidad de $15,00 \pm 0,01 \mathrm{~cm}$. 
g) Porcentaje de sólidos solubles totales ( ${ }^{\circ}$ Brix): se consiguió a partir de un macerado de la pulpa y la placenta del fruto, en 20 frutos por cada repetición y categoría de calidad y se obtuvo el promedio. La medida se logró a través de un refractómetro manual marca BOECO, con una capacidad de $0-32,0 \pm 0,2{ }^{\circ}$ Brix.

h) Peso del fruto (g): se midió el peso total de los frutos producidos por repetición y categoría de calidad, esta se dividió entre el número total de frutos obtenidos. Se determinó por medio de una balanza electrónica marca OCONY UWE HGM20 , con una capacidad de $20000 \pm 1 \mathrm{~g}$.

i) Rendimiento $(\mathrm{kg} / \mathrm{m} 2)$ : este se estimó a partir del peso de la producción obtenida por parcela y la densidad de siembra para cada categoría de calidad. Se consideró como rendimiento comercial la suma de las categorías de primera y segunda calidad y como rendimiento total la suma de las tres categorías de calidad.
Cabe mencionar que se utilizó un diseño experimental irrestricto al azar con cuatro tratamientos (genotipos) y cuatro repeticiones. Cada parcela contó con ocho plantas (dos sacos) y todos los datos se obtuvieron a partir de los frutos totales producidos en dicha parcela. En cuanto a las variables cuantitativas, se realizó un análisis estadístico de variancia y se utilizó la prueba de LSD Fisher con una significancia de $5 \%$ para determinar diferencias entre los tratamientos.

\section{Resultados y discusión}

\section{Edad al inicio de floración y cosecha}

En el cuadro 4 se muestran los resultados de edad al inicio de floración y al inicio de cosecha, para los genotipos de pepino evaluados. El Arioso fue un poco más precoz que los demás genotipos pues inició la floración a los 19 ddt y la cosecha a los $28 \mathrm{ddt}$, mientras que los demás iniciaron la floración a los 21 ddt y la cosecha a los 30 ddt.

Cuadro 4. Edad al inicio de floración y al inicio de cosecha de genotipos de pepino.

\begin{tabular}{|l|l|l|}
\hline Genotipo & Edad al inicio de floración (ddt) & Edad al inicio de cosecha (ddt) \\
\hline Arioso & 19 & 28 \\
\hline Paisaje & 21 & 30 \\
\hline Kalunga & 21 & 30 \\
\hline Cumlaude & 21 & 30 \\
\hline & & \\
\hline Fuente: elaboración propia, a partir de los datos obtenidos. & \\
\hline
\end{tabular}

Según los investigadores incluidos en el cuadro 5, la edad al inicio de cosecha oscila entre 31 y 91 ddt para pepino tipo largo; en este caso, los resultados obtenidos fueron menores a dicho rango, es decir, estos genotipos mostraron una producción más precoz.

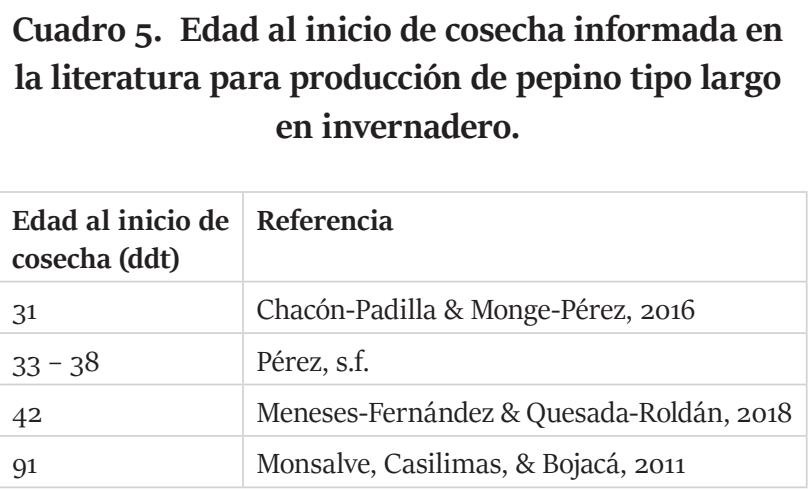

Fuente: elaboración propia, a partir de los datos de la literatura. 
Por consiguiente, la precocidad pudo haber sido inducida por efecto de la alta temperatura y la alta radiación en la que se desarrolló el cultivo, lo cual pudo haber ocasionado un aceleramiento en el metabolismo de las plantas. Los distintos valores de edad al inicio de la cosecha de pepino entre diferentes sitios o épocas de siembra se deben generalmente a las diferencias en la temperatura dentro del invernadero, debido a que las bajas temperaturas provocan una disminución en el ritmo de crecimiento de la planta y un retraso en la maduración del fruto, mientras que una mayor temperatura acelera ambos procesos (Grijalva, Macías, Grijalva, \& Robles, 2011).

\section{Presencia de espinas}

Los resultados para la presencia de espinas en los frutos de los genotipos de pepino se muestran en el cuadro 6 .

\section{Cuadro 6. Presencia de espinas en el fruto de genotipos de pepino.}

\begin{tabular}{|l|l|l|}
\hline Genotipo & Presencia de espinas & Color de espinas \\
\hline Arioso & Intermedio & Blanco \\
\hline Cumlaude & Ausencia & No aplica \\
\hline Kalunga & Ausencia & No aplica \\
\hline Paisaje & Ausencia & No aplica \\
\hline
\end{tabular}

Fuente: elaboración propia, a partir de los datos obtenidos.

En relación a los genotipos de pepino evaluados, todos mostraron frutos sin espinas, con excepción de Arioso (pepino tipo japonés), el cual presentó una cantidad intermedia de espinas y cuyo fruto es largo, con una cáscara dura y brillante que posee muchas espinas blancas, además, es muy susceptible a daños mecánicos.

Básicamente, la presencia de espinas está asociada al tipo de cáscara de cada genotipo. En cuanto a los pepinos tipo largo (holandés) son de cáscara suave y delgada, con estrías o arrugas levemente marcadas y no poseen espinas. En el caso del genotipo Arioso, es un pepino tipo asiático (japonés), los cuales tienen una cáscara gruesa y presentan muchas espinas (Johnny's Selected Seeds, 2014).

\section{Longitud del fruto}

En el cuadro 7 se presentan los resultados de longitud del fruto de los genotipos evaluados.

\section{Cuadro 7. Longitud del fruto de genotipos de pepino.}

\begin{tabular}{|c|c|c|c|c|}
\hline \multirow{2}{*}{ Genotipo } & \multicolumn{4}{|c|}{$\begin{array}{l}\text { Longitud del fruto }(\mathrm{cm}) \text {, según categoría de } \\
\text { calidad }\end{array}$} \\
\hline & Total & Primera & Segunda & Rechazo \\
\hline Kalunga & $31,02 \mathrm{a}$ & $32,57 \mathrm{a}$ & $30,89 \mathrm{a}$ & 29,60 a \\
\hline Arioso & $31,44 \mathrm{~b}$ & $32,76 \mathrm{a}$ & $30,75 \mathrm{a}$ & $30,79 \mathrm{~b}$ \\
\hline Cumlaude & $31,95 \mathrm{c}$ & $33,79 \mathrm{~b}$ & $31,13 \mathrm{a}$ & $30,93 \mathrm{~b}$ \\
\hline Paisaje & $32,38 \mathrm{~d}$ & $34,16 \mathrm{~b}$ & $31,96 \mathrm{~b}$ & $31,03 \mathrm{~b}$ \\
\hline \multicolumn{5}{|c|}{$\begin{array}{l}\text { Categoría de } \\
\text { calidad }\end{array}$} \\
\hline Primera & $33,32 \mathrm{c}$ & & & \\
\hline Segunda & $31,18 \mathrm{~b}$ & & & \\
\hline Rechazo & $30,59 a$ & & & \\
\hline
\end{tabular}

Fuente: elaboración propia, a partir de los datos obtenidos. Nota: Valores con una letra en común no son significativamente diferentes, según prueba LSD Fisher ( $\mathrm{p} \leq \mathrm{0}, 05)$.

Por su parte, el genotipo Paisaje presentó la mayor longitud del fruto con $32,38 \mathrm{~cm}$, mientras que Kalunga obtuvo la menor longitud (31,02 cm); Arioso y Cumlaude presentaron valores intermedios para esta característica. Además, en términos generales, los frutos de primera calidad mostraron una mayor longitud que los de segunda calidad y de rechazo y los de segunda calidad obtuvieron también una mayor longitud que los de rechazo.

De acuerdo con los autores mencionados en el cuadro 8 , la longitud del fruto varía entre 25,91 y 39,30 $\mathrm{cm}$ para pepino tipo largo; a su vez, los resultados obtenidos en la investigación se ubicaron dentro de dicho rango. 
Cuadro 8. Longitud del fruto informada en la literatura para producción de pepino tipo largo en invernadero.

\begin{tabular}{|l|l|}
\hline Longitud del fruto $\mathbf{( c m})$ & Referencia \\
\hline $25,91-37,08$ & Lamb, Shaw, \& Cantliffe, 2001 \\
\hline $26,30-39,30$ & $\begin{array}{l}\text { Shaw, Cantliffe, Rodríguez, Taylor, } \\
\text { \& Spencer, 20oo }\end{array}$ \\
\hline $30,73-36,83$ & $\begin{array}{l}\text { Hochmuth, León, \& Hochmuth, } \\
1996\end{array}$ \\
\hline $31,58-35,77$ & $\begin{array}{l}\text { Chacón-Padilla \& Monge-Pérez, } \\
2016\end{array}$ \\
\hline $32,10-33,40$ & $\begin{array}{l}\text { Jasso-Chaverria, Hochmuth, } \\
\text { Hochmuth, \& Sargent, 2005 }\end{array}$ \\
\hline
\end{tabular}

Fuente: elaboración propia, a partir de los datos de la literatura.

En comparación con otro ensayo realizado en la EEAFBM en la época seca (Chacón-Padilla \& Monge-Pérez, 2016), en el presente trabajo los cuatro genotipos evaluados mostraron una menor longitud del fruto.

\section{Diámetro del fruto}

En el cuadro 9, se presentan los resultados de diámetro del fruto de los genotipos evaluados.

\section{Cuadro 9. Diámetro del fruto de genotipos de pepino.}

\begin{tabular}{|l|l|l|l|l|}
\hline \multirow{2}{*}{ Genotipo } & \multicolumn{4}{|l|}{$\begin{array}{l}\text { Diámetro del fruto (mm), según categoría de } \\
\text { calidad }\end{array}$} \\
\cline { 2 - 5 } & Total & Primera & Segunda & Rechazo \\
\hline Paisaje & $48,74 \mathrm{a}$ & $50,19 \mathrm{ab}$ & $48,09 \mathrm{a}$ & $47,94 \mathrm{~b}$ \\
\hline Kalunga & $49,06 \mathrm{ab}$ & $49,60 \mathrm{a}$ & $49,38 \mathrm{~b}$ & $48,21 \mathrm{~b}$ \\
\hline Cumlaude & $49,34 \mathrm{~b}$ & $50,89 \mathrm{~b}$ & $50,32 \mathrm{~b}$ & $46,80 \mathrm{a}$ \\
\hline Arioso & $52,31 \mathrm{c}$ & $53,32 \mathrm{c}$ & $51,61 \mathrm{c}$ & $51,98 \mathrm{c}$ \\
\hline $\begin{array}{l}\text { Categoría } \\
\text { de calidad }\end{array}$ & & & & \\
\hline Primera & $51,00 \mathrm{c}$ & & & \\
\hline Segunda & $49,85 \mathrm{~b}$ & & & \\
\hline Rechazo & $48,73 \mathrm{a}$ & & & \\
\hline
\end{tabular}

Fuente: elaboración propia, a partir de los datos obtenidos. Nota: valores con una letra en común no son significativamente diferentes, según prueba LSD Fisher ( $\mathrm{p} \leq \mathrm{0,05})$.
El genotipo Arioso alcanzó el mayor valor para esta característica con 52,31 mm, mientras que Paisaje obtuvo el menor valor (48,74 mm) y Kalunga y Cumlaude alcanzaron valores intermedios. Por otra parte, a nivel general, los frutos de primera calidad presentaron un mayor diámetro que los de segunda calidad y de rechazo; asimismo, los de segunda calidad mostraron un mayor diámetro que los de rechazo.

Según los investigadores que se indican en el cuadro 10, el diámetro del fruto oscila entre 43,00 y 53,30 mm para pepino tipo largo; en el caso de los resultados hallados en el presente ensayo, se ubicaron dentro de dicho rango.

Cuadro 10. Diámetro del fruto informado en la literatura para producción de pepino tipo largo en invernadero.

\begin{tabular}{|l|l|}
\hline Diámetro del fruto $(\mathbf{m m})$ & Referencia \\
\hline $43,00-52,00$ & $\begin{array}{l}\text { Shaw, Cantliffe, Rodríguez, Taylor, \& } \\
\text { Spencer, 2000 }\end{array}$ \\
\hline 44,00 & $\begin{array}{l}\text { Jasso-Chaverria, Hochmuth, Hochmuth, } \\
\text { \& Sargent, 2005 }\end{array}$ \\
\hline $46,60-50,09$ & Chacón-Padilla \& Monge-Pérez, 2016 \\
\hline $48,30-53,30$ & Hochmuth, León, \& Hochmuth, 1996 \\
\hline 50,80 & Lamb, Shaw, \& Cantliffe, 2001 \\
\hline
\end{tabular}

Fuente: elaboración propia, a partir de los datos de la literatura.

\section{Número de frutos por planta}

Los datos de número de frutos por planta se presentan en el cuadro 11.

\section{Cuadro 11. Número de frutos por planta de genotipos de pepino.}

\begin{tabular}{|l|l|l|l|l|}
\hline \multirow{2}{*}{ Genotipo } & \multicolumn{4}{|l|}{$\begin{array}{l}\text { Número de frutos por planta, según categoría } \\
\text { de calidad }\end{array}$} \\
\cline { 2 - 5 } & Total & Primera & Segunda & Rechazo \\
\hline Arioso & $16,60 \mathrm{a}$ & $5,75 \mathrm{a}$ & $5,47 \mathrm{a}$ & $5,38 \mathrm{a}$ \\
\hline Paisaje & $24,41 \mathrm{~b}$ & $7,91 \mathrm{~b}$ & $8,47 \mathrm{~b}$ & $8,03 \mathrm{~b}$ \\
\hline Cumlaude & $24,84 \mathrm{~b}$ & $8,83 \mathrm{bc}$ & $8,38 \mathrm{~b}$ & $7,63 \mathrm{~b}$ \\
\hline Kalunga & $26,92 \mathrm{c}$ & $9,13 \mathrm{c}$ & $9,42 \mathrm{c}$ & $8,37 \mathrm{~b}$ \\
\hline
\end{tabular}

Fuente: elaboración propia, a partir de los datos obtenidos. Nota: Valores con una letra en común no son significativamente diferentes, según prueba LSD Fisher ( $\mathrm{p} \leq \mathrm{0}, 05)$. 
En este caso, el genotipo Kalunga fue el que presentó la mayor producción total (26,92 frutos por planta). Por otra parte, Arioso fue el que presentó la menor cantidad de frutos de primera calidad, con 5,75 frutos por planta y la menor producción total de frutos (16,6o frutos por planta). A su vez, Paisaje y Cumlaude obtuvieron valores intermedios para esta característica.

De acuerdo con los autores que se mencionan en el cuadro 12, la producción total de frutos varía entre 8,00 y 24,oo frutos por planta para pepino tipo largo. En esta investigación, los resultados se ubicaron por encima de dicho rango, con excepción del genotipo Arioso, lo cual demuestra una mayor prolificidad en este caso para los genotipos Paisaje, Cumlaude y Kalunga.

\section{Cuadro 12. Número de frutos por planta informado en la literatura para producción de pepino tipo largo en invernadero.}

\begin{tabular}{|l|l|}
\hline $\begin{array}{l}\text { Número de frutos por } \\
\text { planta }\end{array}$ & Referencia \\
\hline $8,00-14,00$ & Crosby, 20o8 \\
\hline $8,70-12,60$ & $\begin{array}{l}\text { Meneses-Fernández \& Quesada-Roldán, } \\
2018\end{array}$ \\
\hline $11,00-15,20$ & Hochmuth, León, \& Hochmuth, 1996 \\
\hline $12,70-23,80$ & $\begin{array}{l}\text { Shaw, Cantliffe, Rodríguez, Taylor, \& } \\
\text { Spencer, 20oo }\end{array}$ \\
\hline $13,00-24,00$ & Lamb, Shaw, \& Cantliffe, 2oo1 \\
\hline $15,03-21,03$ & Chacón-Padilla \& Monge-Pérez, 2016 \\
\hline $20,30-23,80$ & Pérez, s.f. \\
\hline
\end{tabular}

Fuente: elaboración propia, a partir de los datos de la literatura.

El pepino tipo largo requiere aproximadamente 15 días para desarrollar cada fruto, a partir de la antesis. Además, en las plantas de pepino evaluadas se observó que solo se produce un fruto por nudo.

Se ha informado que la incidencia de alta temperatura (mayor a $30{ }^{\circ} \mathrm{C}$ ) durante la producción de pepino provoca desequilibrios en las plantas, dando lugar a malformaciones de hojas y frutos defectuosos (Grijalva, Macías, Grijalva, \& Robles, 2011). Esto probablemente se presentó en el presente ensayo, donde las temperaturas máximas dentro del invernadero alcanzaron inclusive los $37,4{ }^{\circ} \mathrm{C}$, lo que pudo haber favorecido la producción de frutos de calidad de rechazo (entre 5,38 y 8,37 frutos por planta).

Es importante mencionar que el genotipo Arioso mostró una alta producción de flores masculinas, lo cual redujo significativamente su producción. Este genotipo presentó en algunos casos solo flores masculinas incluso en cuatro nudos consecutivos y hasta el siguiente nudo reveló una flor femenina.

Cabe destacar que existen varias condiciones ambientales estresantes que pueden influir en la proporción de flores masculinas y femeninas producidas en plantas de pepino, como la densidad de las plantas, la baja intensidad de luz y la temperatura (Schultheis, y otros, 2000). Por lo tanto, a altas densidades de siembra las plantas se pueden estresar por la competencia por agua, nutrientes o luz y esto puede causar un aumento en el número de flores masculinas. Por otra parte, una combinación de condiciones de poca luz y alta temperatura también pueden reducir el número de flores femeninas (Schultheis, y otros, 2000).

Por lo tanto, los factores endógenos que determinan la aparición de un tipo de flor tienen relación con los niveles de reguladores de crecimiento como las auxinas y giberelinas, entre otras. Además, niveles altos de nitrógeno retrasan la aparición de flores femeninas e igualmente la relación N/K es de gran influencia en la aparición de una u otro tipo de flores (Reche, 2011).

Otros investigadores reportaron, en la época seca en la EEAFBM, entre 15,03 y 21,03 frutos totales por planta al evaluar varios genotipos de pepino tipo largo (ChacónPadilla \& Monge-Pérez, 2016), lo que representa valores inferiores a los obtenidos en el presente trabajo (excepto para el genotipo Arioso). En efecto, esto parece sugerir que en la época lluviosa se produce una mayor cantidad de frutos por planta en los pepinos tipo largo cultivados bajo invernadero, en comparación con la época seca.

\section{Peso del fruto}

En el cuadro 13 se presentan los datos de peso del fruto de los genotipos evaluados. 
Cuadro 13. Peso del fruto de genotipos de pepino.

\begin{tabular}{|l|l|l|l|l|}
\hline \multirow{2}{*}{ Genotipo } & \multicolumn{4}{|l|}{ Peso del fruto (g), según categoría de calidad } \\
\cline { 2 - 5 } & Total & Primera & Segunda & Rechazo \\
\hline Kalunga & $470,17 \mathrm{a}$ & $464,05 \mathrm{a}$ & $474,27 \mathrm{ab}$ & $472,20 \mathrm{~b}$ \\
\hline Arioso & $478,00 \mathrm{a}$ & $529,05 \mathrm{C}$ & $472,17 \mathrm{a}$ & $432,78 \mathrm{a}$ \\
\hline Paisaje & $493,66 \mathrm{~b}$ & $497,20 \mathrm{~b}$ & $483,89 \mathrm{~b}$ & $499,90 \mathrm{C}$ \\
\hline Cumlaude & $517,77 \mathrm{C}$ & $548,98 \mathrm{~d}$ & $502,40 \mathrm{C}$ & $501,92 \mathrm{C}$ \\
\hline
\end{tabular}

Fuente: elaboración propia, a partir de los datos obtenidos. Nota: Valores con una letra en común no son significativamente diferentes, según prueba LSD Fisher ( $\mathrm{p} \leq \mathrm{0,05})$.

En referencia al genotipo Cumlaude, este presentó los frutos con el mayor peso total (517,77 g) y Kalunga y Arioso produjeron los frutos con el menor valor para esta característica (entre 470,17 y 478,oo g). Asimismo, Cumlaude alcanzó el mayor peso del fruto en cada una de las categorías de calidad.

Según los investigadores que se indican en el cuadro 14, el peso del fruto oscila entre 278,00 y 616,90 g para pepino tipo largo. En cuanto a los resultados hallados en el presente ensayo, se ubicaron dentro de dicho rango.

\section{Cuadro 14. Peso del fruto informado en la literatura para producción de pepino tipo largo en invernadero.}

\begin{tabular}{|l|l|}
\hline Peso del fruto (g) & Referencia \\
\hline $278,00-342,00$ & Crosby, 2008 \\
\hline $294,84-416,74$ & Lamb, Shaw, \& Cantliffe, 2001 \\
\hline $295,00-518,00$ & $\begin{array}{l}\text { Shaw, Cantliffe, Rodríguez, Taylor, \& } \\
\text { Spencer, 20oo }\end{array}$ \\
\hline $313,00-365,00$ & $\begin{array}{l}\text { Grijalva, Macías, Grijalva, \& Robles, } \\
2011\end{array}$ \\
\hline $439,75-480,82$ & $\begin{array}{l}\text { Chacón-Padilla \& Monge-Pérez, } \\
2016\end{array}$ \\
\hline $449,06-616,90$ & Hochmuth, León, \& Hochmuth, 1996 \\
\hline $458,62-493,91$ & $\begin{array}{l}\text { Meneses-Fernández \& Quesada- } \\
\text { Roldán, 2018 }\end{array}$ \\
\hline
\end{tabular}

Fuente: elaboración propia, a partir de los datos de la literatura.

\section{Rendimiento}

En el cuadro 15 se presentan los datos de rendimiento de los genotipos evaluados.

\section{Cuadro 15. Rendimiento de genotipos de pepino.}

\begin{tabular}{|l|l|l|l|l|l|}
\hline \multirow{2}{*}{ Genotipo } & \multicolumn{5}{|c|}{ Rendimiento (kg/m2), según categoría de calidad } \\
\cline { 2 - 7 } & Total & Comercial & Primera & Segunda & Rechazo \\
\hline Arioso & 20,49 a & $14,47 \mathrm{a}$ & $7,80 \mathrm{a}$ & $6,67 \mathrm{a}$ & $6,02 \mathrm{a}$ \\
\hline Paisaje & $31,26 \mathrm{~b}$ & $20,84 \mathrm{~b}$ & $10,19 \mathrm{~b}$ & $10,65 \mathrm{~b}$ & $10,42 \mathrm{~b}$ \\
\hline Kalunga & $\begin{array}{l}32,75 \\
\text { bc }\end{array}$ & $22,65 \mathrm{bc}$ & $11,02 \mathrm{~b}$ & $11,63 \mathrm{c}$ & $10,10 \mathrm{~b}$ \\
\hline Cumlaude & $33,46 \mathrm{c}$ & $23,53 \mathrm{c}$ & $12,61 \mathrm{c}$ & $10,92 \mathrm{bc}$ & $9,93 \mathrm{~b}$ \\
\hline
\end{tabular}

Fuente: elaboración propia, a partir de los datos obtenidos. Nota: Valores con una letra en común no son significativamente diferentes, según prueba LSD Fisher ( $\mathrm{p} \leq \mathrm{0}, 05)$.

Se infiere que el genotipo que alcanzó el menor rendimiento, tanto por cada categoría de calidad, como a nivel total y comercial, fue Arioso. Por otra parte, Cumlaude presentó el mayor rendimiento en la categoría de primera calidad $\left(12,61 \mathrm{~kg} / \mathrm{m}^{2}\right)$, así como a nivel comercial y total con 23,53 y $33,46 \mathrm{~kg} / \mathrm{m}^{2}$ respectivamente.

De acuerdo con los investigadores que se mencionan en el cuadro 16 , el rendimiento oscila entre 1,80 y $25,90 \mathrm{~kg} /$ $\mathrm{m}^{2}$ para pepino tipo largo; los resultados hallados en este ensayo fueron superiores a dicho rango, es decir, más productivos, con excepción de Arioso.

\section{Cuadro 16. Rendimiento informado en la literatura para producción de pepino tipo largo en invernadero.}

\begin{tabular}{|l|l|}
\hline Rendimiento $\left.\mathbf{( k g} / \mathbf{m}^{2}\right)$ & Referencia \\
\hline $1,80-15,50$ & Grijalva, Macías, Grijalva, \& Robles, 2011 \\
\hline $8,10-8,50$ & Monsalve, Casilimas, \& Bojacá, 2011 \\
\hline $8,80-12,50$ & Pérez, s.f. \\
\hline $9,36-16,03$ & Hochmuth, León, \& Hochmuth, 1996 \\
\hline $10,37-15,57$ & $\begin{array}{l}\text { Meneses-Fernández \& Quesada-Roldán, } \\
2018\end{array}$ \\
\hline $18,03-25,90$ & Chacón-Padilla \& Monge-Pérez, 2016 \\
\hline
\end{tabular}

Fuente: elaboración propia, a partir de los datos de la literatura. 
De acuerdo con una investigación realizada en el invernadero de la EEAFBM, se reportó un rendimiento total entre 10,37 y $15,57 \mathrm{~kg} / \mathrm{m}^{2}$ en pepino tipo largo de la variedad fuerte (Meneses-Fernández \& Quesada-Roldán, 2018), lo cual es inferior al rendimiento total obtenido en la presente investigación para todos los genotipos evaluados.

En comparación con los datos obtenidos en otro estudio desarrollado en el invernadero de la EEAFBM en la época seca (Chacón-Padilla \& Monge-Pérez, 2016), en la presente investigación (época lluviosa) el rendimiento comercial y total fue mayor para cada uno de los cuatro genotipos evaluados, lo que parece sugerir que la temporada de lluvias brinda mejores condiciones climáticas dentro del invernadero para lograr una mayor producción en este tipo de pepino.

\section{Porcentaje de sólidos solubles totales}

En el cuadro 17 se presentan los resultados de porcentaje de sólidos solubles totales del fruto de los genotipos evaluados.

\section{Cuadro 17. Porcentaje de sólidos solubles totales del fruto de genotipos de pepino.}

\begin{tabular}{|c|c|c|c|c|}
\hline \multirow[t]{2}{*}{ Genotipo } & \multicolumn{4}{|c|}{$\begin{array}{l}\text { Porcentaje de sólidos solubles totales ( }{ }^{\circ} \text { Brix), } \\
\text { según categoría de calidad }\end{array}$} \\
\hline & Total & Primera & Segunda & Rechazo \\
\hline Kalunga & $3,01 \mathrm{a}$ & 2,99 a & $3,02 \mathrm{a}$ & $3,03 \mathrm{a}$ \\
\hline Cumlaude & 3,03 a & $3,07 \mathrm{ab}$ & $3,05 \mathrm{a}$ & $2,98 \mathrm{a}$ \\
\hline Paisaje & $3,09 \mathrm{~b}$ & $3,12 \mathrm{~b}$ & $3,10 \mathrm{a}$ & $3,07 \mathrm{a}$ \\
\hline Arioso & $3,64 \mathrm{c}$ & $3,79 \mathrm{c}$ & $3,82 \mathrm{~b}$ & $3,31 \mathrm{~b}$ \\
\hline \multicolumn{5}{|l|}{$\begin{array}{l}\text { Categoría de } \\
\text { calidad }\end{array}$} \\
\hline Primera & $3,24 \mathrm{~b}$ & & & \\
\hline Segunda & $3,25 \mathrm{~b}$ & & & \\
\hline Rechazo & $3,10 \mathrm{a}$ & & & \\
\hline \multicolumn{5}{|c|}{$\begin{array}{l}\text { Fuente: elaboración propia, a partir de los datos obtenidos. Nota } \\
\text { Valores con una letra en común no son significativamente diferentes } \\
\text { según prueba LSD Fisher ( } \mathrm{p} \leq 0,05) \text {. }\end{array}$} \\
\hline
\end{tabular}

términos generales, los frutos de primera y segunda calidad alcanzaron un mayor valor que los de rechazo para esta cualidad.

Unos investigadores hallaron en varios genotipos de pepino tipo largo cultivados en invernadero, valores para esta característica entre 3,38 y 3,67 ${ }^{\circ}$ Brix (Chacón-Padilla \& Monge-Pérez, 2016); los valores obtenidos en el presente estudio son menores a dicho rango, con excepción del resultado encontrado para Arioso.

\section{Conclusiones}

Se logró determinar que los frutos de pepino de primera calidad mostraron mayor longitud y diámetro que los de segunda calidad y de rechazo. En términos generales, los frutos de primera y segunda calidad obtuvieron un mayor porcentaje de sólidos solubles totales que los de calidad de rechazo.

En cuanto al genotipo Cumlaude, alcanzó el mayor rendimiento de primera calidad $\left(12,61 \mathrm{~kg} / \mathrm{m}^{2}\right)$, por lo que se considera el mejor adaptado a las condiciones en que se desarrolló la investigación; por el contrario, Arioso obtuvo el menor rendimiento comercial y total.

\section{Agradecimientos}

Los autores agradecen el financiamiento recibido por parte de CONARE, así como de la Universidad de Costa Rica para la realización de este trabajo. Asimismo, agradecen la colaboración de Ana Julia Sánchez, Karla Chacón, Ana Rebeca Zeledón, Carolina Ramírez, Werner Salazar, Julio Loáiciga, Julio Vega, Andrés Oviedo y Carlos González en el trabajo de campo, además, Mario Monge en la revisión de la traducción del resumen al idioma inglés. 


\section{Referencias}

Bojacá, C., \& Monsalve, O. (2012). Manual de producción de pepino bajo invernadero. Bogotá, Colombia: Universidad de Bogotá Jorge Tadeo Lozano.

Casaca, A. (2005). Guías tecnológicas de frutas y vegetales. El cultivo del pepino. http://www.innovacion.gob. sv/inventa/attachments/article/2286/pepino.pdf

Centro de Exportación e Inversión de la República Dominicana. (2008). Perfil económico del pepino (Cucumis sativus L.). http://cei-rd.gov. do/estudios_economicos/estudios_productos/ perfiles/PEPINO.pdf

Chacón-Padilla, K., \& Monge-Pérez, J. E. (2016). Evaluación del rendimiento y la calidad de seis genotipos de pepino (Cucumis sativus L.) cultivados bajo invernadero en Costa Rica. Revista Colombiana de Ciencias Hortícolas, 10(2), 323-332.

Crosby, L. C. (2008). Growth and consumer evaluation of Cucumis sativus L. cultivated in controlled environments. Tesis de maestría en Horticultura, Texas Tech University.

FAO. (2002). El cultivo protegido en clima mediterráneo: pepino. http://www.fao.org/3/s863os/s863os.pdf

FAO. (2015). Estadísticas de producción de pepino. http:// faostat3.fao.org/browse/T/TP/S

Grijalva, R. L., Macías, R., Grijalva, S. A., \& Robles, F. (2011). Evaluación del efecto de la fecha de siembra en la productividad y calidad de híbridos de pepino europeo bajo condiciones de invernadero en el noroeste de Sonora. Biotecnia, 13(1), 29-36.

Haifa. (2011). Nutritional recommendations for cucumber in open fields, tunnels and greenhouse. http:// www.haifa-group.com/files/Guides/Cucumber. pdf
Hochmuth, R. C., León, L. L., \& Hochmuth, G. J. (1996). Evaluation of twelve greenhouse cucumber cultivars and two training systems over two seasons in Florida. Proceedings of the Florida State Horticultural Society, 109(1), 174-177.

Jasso-Chaverria, C., Hochmuth, G. J., Hochmuth, R. C., \& Sargent, S. A. (2005). Fruit yield, size, and color responses of two greenhouse cucumber types to nitrogen fertilization in perlite soilless culture. Hort Technology, 15(3), 565-571.

Johnny's Selected Seeds. (2014). Cucumber types and terminology. http://www.johnnyseeds. com/assets/information/cucumbers-typesterminology-8989.pdf

Kapuriya, V. K., Ameta, K. D., Teli, S. K., Chittora, A., Gathala, S., \& Yadav, S. (2017). Effect of spacing and training on growth and yield of polyhouse grown cucumber (Cucumis sativus L.). International Journal of Current Microbiology and Applied Sciences, 6(8), 299-304.

Lamb, E. M., Shaw, N. L., \& Cantliffe, D. J. (2001). Beit Alpha cucumber: a new greenhouse crop for Florida. http://www.hos.ufl.edu/protectedag/ EDIS/CV27700.pdf

López-Elías, J., Garza, S., Huez, M. A., Jiménez, J., Rueda, E. O., \& Murillo, B. (2015). Producción de pepino (Cucumis sativus L.) en función de la densidad de plantación en condiciones de invernadero. European Scientific Journal, 11(24), 25-36.

Marín, F. (2010). Cuantificación y valoración de estructuras y procesos de producción agrícola bajo ambientes protegidos en Costa Rica. http://www.mag.go.cr/ bibliotecavirtual/aoo2go.pdf

Meneses-Fernández, C., \& Quesada-Roldán, G. (2018). Crecimiento y rendimiento del pepino holandés en ambiente protegido y con sustratos orgánicos alternativos. Agronomía Mesoamericana, 29(2), 235-250. 
Monge-Pérez, J. E. (2016). Evaluación preliminar de 201 genotipos de ocho diferentes hortalizas (berenjena, chile dulce, zucchini, ayote, sandía, pepino, tomate y melón) cultivados bajo invernadero en Costa Rica. Em E. Solano, La investigación en Guanacaste II (pp. 277-300). San José, Costa Rica: Nuevas Perspectivas.

Monsalve, O. I., Casilimas, H. A., \& Bojacá, C. R. (2011). Evaluación técnica y económica del pepino y el pimentón como alternativas al tomate bajo invernadero. Revista Colombiana de Ciencias Hortícolas, 5(1), 69-82.

Mora, N., Campos, H., Fernández, J. F., \& Morales, S. (2007). Caracterización de la agrocadena pepino holandés. Alajuela, Costa Rica: Dirección Regional Central Occidental, Ministerio de Agricultura y Ganadería.

Musmade, A., \& Desai, U. (2003). Tratado de ciencia y tecnología de las hortalizas: el pepino y el melón. Zaragoza, España: Acribia.

Pérez, M. (s.f.). Productividad de variedades de pepino europeo (Cucumis sativus L.) bajo cultivo hidropónico en malla y multitúnel. https:// www.intagri.com/articulos/horticulturaprotegida/productividad-variedades-de-pepinoeuropeo\#sthash.ZtqP3aig.dpbs

Reche, J. (2011). Cultivo de pepino en invernadero. Madrid, España: Ministerio de Medio Ambiente y Medio Rural y Marino.

Rozano, V., Quiróz, C., Acosta, J. C., Pimentel, L. A., \& Quiñones, E. I. (2004). Hortalizas, las llaves de la energía. Revista Digital Universitaria, 6(9), 1-30.

Sarhan, T. Z., \& Ismael, S. F. (2014). Effect of low temperature and seaweed extracts on flowering and yield of two cucumber cultivars (Cucumis sativus L.). International Journal of Agricultural and Food Research, 3(1), 41-54.
Schultheis, J., Averre, C., Boyette, M., Estes, E., Holmes, G., Monks, D., \& Sorensen, K. (2000). Commercial production of pickling and slicing cucumbers in North Carolina. https://content.ces.ncsu.edu/ commercial-production-of-pickling-and-slicingcucumbers-in-north-carolina

Sediyama, M. A., Nascimento, J. L., Lopes, I. P., Lima, P. C., \& Vidigal, S. M. (2014). Tipos de poda em pepino dos grupos aodai, japonés e caipira. Horticultura Brasileira, 32(4), 491-496.

Shaw, N. L., Cantliffe, D. J., Rodríguez, J. C., Taylor, S., \& Spencer, D. M. (2000). Beit Alpha cucumber: an exciting new greenhouse crop. Proceedings of the Florida State Horticultural Society, 113(1), 247-253.

Torres, S. (2007). Valoración agronómica de dos variedades de mostaza (Brassica juncea), pepino (Cucumis sativus) y rábano (Raphanus sativus). Guácimo, Costa Rica: Escuela de Agricultura de la Región Tropical Húmeda.

Valenciano, J. A., Salas, A. M., \& Díaz, R. (2013). Sistemas de financiamiento en cadenas agrícolas rurales: un caso no exitoso en la producción de pepino en Zarcero, Costa Rica. Revista ABRA, 33(46), 13-29.

Vasco, R. (2003). El cultivo de pepino bajo invernadero. En F. A. Camacho, Técnicas de producción en cultivos protegidos (Vol. 2, pp. 691-722). Madrid, España: Caja Rural Intermediterránea (Cajamar). 